\title{
Prevalência da microbiota no trato digestivo de fêmeas de Lutzomyia longipalpis (Lutz \& Neiva, 1912) (Diptera: Psychodidae) provenientes do campo
}

\author{
Prevalence of the microbiota in the digestive tract of wild-caught females \\ of Lutromyia longipalpis (Lutz \& Neiva) (Diptera: Psychodidae)
}

\section{Sandra Maria Pereira de Oliveira', Bianca Aguiar de Moraes², Claudia Abrantes Gonçalves', Cristina Maria Giordano-Dias', José Mario d'Almeida ${ }^{3}$, Marise Dutra Asensi² Rubens Pinto Mello e Reginaldo Peçanha Brazil ${ }^{4}$}

\begin{abstract}
Resumo No presente trabalho foram dissecados o trato digestivo de 245 fêmeas de Lutzomyia longipalpis originários da Gruta da Lapinha, Município de Lagoa Santa, MG, formando 7 grupos de 35 flebotomíneos. Das 8 espécies de bactérias isoladas houve uma predominância de bacterias Gram negativas (BGN) pertencentes ao grupo de não fermentadoras de açúcar das seguintes espécies: Acinetobacter lowffii, Stenotrophomonas maltophhilia, Pseudomonas putida e Flavimonas orizihabitans. No grupo das fermentadoras tivemos: Enterobacter cloacae e Klebsiella ozaenae. No grupo dos Gram positivos foram identificados Bacillus thuringiensis e Staphylococcus spp.
\end{abstract}

Palavras-chaves: Lutzomyia longipalpis. Flebotomíneos. Bactérias.

\begin{abstract}
We dissected the digestive tract of 245 females in pools of 35 flies forming 7 groups. These flies were Lutzomyia longipalpis originating from Lapinha Cave, Lagoa Santa, Minas Gerais. Out of the 8 species of bacteria isolated there was a predominancy of Gram negative bacterias (GNB) in the group of non-fermenters of sugar belonging to the following species: Acinetobacter Iwoffii, Stenotrophomonas maltophilia, Pseudomonas putida and Flavimonas orizihabitans. The group of GNB fermenters were: Enterobacter cloacae and Klebsiella ozaenae. In the Gram positive group we isolated the genera Bacillus thuringiensis and Staphylococcus spp.
\end{abstract}

Key-words: Lutzomyia longipalpis. Sandflies. Bacterias.

Bactérias têm sido identificadas no trato digestivo de várias espécies de insetos ${ }^{32}$, e em algumas espécies, elas podem interferir no desenvolvimento de parasitos de importância médica transmitidos por insetos ${ }^{426}$. Em insetos criados em laboratório a presença de bactérias no trato digestivo ocorre com mais freqüência, acarretando alto índice de mortalidade ${ }^{12914}$.
Flebotomíneos em condições naturais, são capazes de controlar um número limitado de bactérias, pois seu mecanismo de defesa é ineficiente contra infecções maciças ${ }^{27}$. Esse mecanismo, não só é responsável por proteger esses vetores contra infecções no intestino, como também é um fator essencial para a susceptibilidade de flebotomíneos em albergar Leishmania spp ${ }^{129}$.

\footnotetext{
1. Departamento de Entomologia, 2. Departamento de Bacteriologia, 3. Departamento de Biologia do Instituto Oswaldo Cruz, Rio de Janeiro, RJ e 4. Centro de Pesquisa René Rachou da FIOCRUZ, Belo Horizonte, MG, Brasil.

Endereço para correspondência: Dr. Reginaldo Peçanha Brazil. Laboratório de Leishmanioses/CPqRR/FIOCRUZ. Av. Augusto de Lima 1715, 30190-002 Belo Horizonte, MG, Brasil.

Fax: 5531 295-3115.

e-mail: rpbrazil@cpqrr.fiocruz.br

Recebido para publicação em 15/3/99.
} 
Em condições naturais, fêmeas de flebotomíneos, além de exercer a hematofagia, alimentam-se de açúcares, derivados de várias fontes (folhas, frutos e secreções de afídeos), onde têm a oportunidade de ingerir microrganismos ${ }^{10} 1728$.

No presente estudo são identificadas bactérias do trato digestivo de fêmeas de Lutzomyia longipalis, na natureza, o que irá subsidiar estudos futuros tanto ao nível de campo como experimental, de laboratório.

L. longipalpis foram capturados com armadilha luminosa tipo CDC, externamente, na Gruta da Lapinha, município de Lagoa Santa, MG e levados ao laboratório no Departamento de Biologia/ Instituto Oswaldo Cruz - FIOCRUZ/RJ sem qualquer tipo de alimento. Para os estudos bacteriológicos foram usados meios de cultura desidratados, da marca DIFCO, adotando-se as instruções, no preparo, distribuição e esterilização, bem como, o Sistema de Identificação de Fermentadores e Não Fermentadores Crystal Enteric (Becton \& Dickinson Microbiology Systems EUA).

Foram, dissecadas 245 fêmeas reunidas em 7 grupos, formando um pool de 35 insetos por grupo, das quais, extraímos o trato digestivo (tubo digestivo e tubos de Malpighi). As fêmeas foram selecionadas e colocadas no congelador, por alguns minutos, para diminuir as atividades vitais e facilitar o manuseio. Posteriormente, foram processadas em câmara de fluxo laminar, submetidos a 1 lavagem em hipoclorito de sódio a $5 \%$ e 2 passagens em água destilada estéril ${ }^{13}$. Ainda na câmara de fluxo laminar, os insetos foram dissecados em salina estéril e os tratos digestivos obtidos mergulhados em caldo simples, onde foram macerados. O material foi semeado em placa de ágar columbia contendo $5 \%$ de sangue desfibrinado de carneiro (agar-sangue); agar xilose-lisina-descarboxilase (AXLD), agar eosinaazul de metileno (AEMB), aAgar hektour (AHK), agar cetrimide e em caldo tioglicolato. Os meios permaneceram em estufa, incubados à temperatura de $37^{\circ} \mathrm{C}$ por 24 a 48 horas. A partir do crescimento bacteriano obtido em caldo tioglicolato, foram realizadas novas semeaduras, seguindo o mesmo procedimento ${ }^{1821}$.

A caracterização primária dos microrganismos foi obtida através dos aspectos gerais das colônias, coloração pelo método de Gram, bem como pelos resultados obtidos nos testes bioquímicos de triagem. Os microrganismos Gram-negativos foram caracterizados utilizando os meios básicos de Costa e Vérnin (CV), meio de sulfeto-indol-motilidade (SIM) e citrato de Simmons ${ }^{22}$.

A identificação das espécies de bactérias baseou-se nos dados das provas bioquímicas, fisiológicas, destacando-se a capacidade de fermentação de açúcares (glicose, sacarose e manitol; produção de descarboxilases (lisina, arginina e ornitina) e desoxiribonuclease (DNAse). Além do Sistema de Identificação de Fermentadores e Não Fermentadores Crystal (BBL) na classificação final57815182122.

Das 8 espécies isoladas do trato digestivo e tubos de Malphighi (Figura 1), foram predominantes os bacilos Gram negativos (BGN) pertencentes ao grupo dos não fermentadores de açúcares, representados por: Acinetobacter Iwoffii, Stenotrophomonas maltophilia, Pseudomonas putida e Flavimonas orizihabitans. O grupo dos BGN, fermentadores foram Enterobacter cloacae e Klebsiella ozaenae. As espécies de bactérias Gram positivas foram: Bacillus tthuringiensis e Staphylococcus spp.

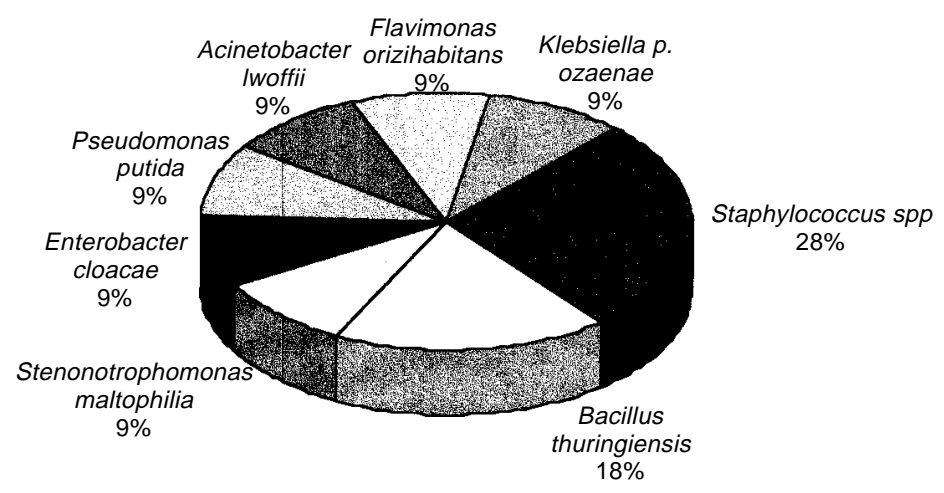

Figura 1 - Espécies bacterianas isoladas de fêmeas de Lutzomyia longipalpis provenientes do campo. 
Com os resultados obtidos, a partir de fêmeas de L. longipalpis capturadas na Gruta da Lapinha, MG, observou-se uma diversidade na população bacteriana nesses flebótomos à semelhança do que fora encontrado em Phlebotomus papatasi no Egito ${ }^{12}$ e em menor número, quando comparada com Musca and Glossina ${ }^{25} 31$.

Dentre as 8 espécies de bactérias isoladas pertencentea a família Enterobacteriaceae, Klebsiella p. ozaenae já foi isolada de mosquitos do gênero Culex, Aedes e Psorophora sendo ela considerada como patógeno ${ }^{11} 16{ }^{30}$. A espécie Enterobacter cloacae também foi identificada em outros insetos ${ }^{32}$ sendo a espécie mais comumente encontrada no intestino de insetos de importância médica, inclusive em flebotomíneos criados em laboratório ${ }^{24}$.

Os bastonetes Gram negativos (BGN), não fermentadores de açúcares, identificados em Lutzomyia longipalpis, representado por Acinetobacter Iwoffii, Stenotrophomonas maltophilia, Pseudomonas putida e Flavimonas orizihabitans, também já foram assinalados por vários autores em diferentes insetos ${ }^{12} 1323$.

A espécie Bacillus thuringiensis (Gram positiva) foi detectada em $18 \%$ das fêmeas dissecadas. Esta espécie também foi isolado do trato digestivo e tubos de Malpighi de machos de Lutzomyia longipalpis, em condições experimentais (observações não publicadas) e em Aedes triseriatus ${ }^{11}$. Bacillus thuringiensis variedade israeliensis (B.t.i.) tem sido um eficiente agente de controle biológico de uma grande variedade de insetos, principalmente, dípteros hematófagos que têm desenvolvimento de formas imaturas em água ${ }^{1920}$. Fêmeas $P$. papatasi alimentandas em diferentes fontes de açúcares tornam-se suscetíveis a ação do (B.t.i. $)^{34}$. Larvas de $P$. papatasi e $L$. longipalpis foram suscetíveis ao B.t.i., cuja toxicidade foi comprovada em adultos de P. papatasi, P. argentipes, P.perniciosus e L. longipalpis ${ }^{33}$.

Espécies não identificadas de Staphylococcus (Gram-positivos) foram encontrado também em P. $t o b b i^{29}$ e em mosca do gênero Cochliomyia ${ }^{6}$. Cocos Gram-positivos foram descritos no trato digestivo de P. papatasi em condições naturais ${ }^{12}$.

Com o presente estudo, é possível admitir as espécies de bacterias dos gêneros: Acinetobacter, Stenotrophomonas, Pseudomonas e Enterobacter, possam fazer parte da flora do trato digestivo de fêmeas de $L$. longipalpis, em condições naturais.

\section{REFERÊNCIAS BIBLIOGRÁFICAS}

1. Adler S, Theodor O. The transmission of Leishmania tropica from artificially infected sandflies to man. Annals of. Tropical Medicine \&. Parasitology 21:88-110, 1927a.

2. Adler S, Theodor O. Attempts to transmit Leishmania tropica by bite: the transmission of L. tropica by Phebotomus sergpti. Annals of Tropical Medicine \&. Parasitology 23:1-18, 1927b

3. Barjac H, Larget I, killick-Kendrick R. Toxicitté de Bacillus thuringiensis var. israelensis, sérotype Hi4, pour les larves de phlébotomes, vecteurs de leishmanioses. Bulletin de la Societé de Pathologie Exotique 74:485-489, 1981.

4. Beier MS, Pumpuni CB, Beier JC, Davis JR. Effects of para-aminobenzoic acid, insulin, and gentamicin on Plasmodium falciparum development in anopheline mosquitoes (Diptera: Culicidae). Journal of Medical Entomology 31:561-565, 1994.

5. Brukcner DA, Colonna P. Nomenclatue for Aerobic and Facultative Bacteria. Clinical Infectious Diseases 25:110, 1997.

6. Caballero M, Hernandez G, Poudevigne F, Ruiz-Martinez I. Isolation and Identification of Bacteria Associated with the Screworm Fly Cochliomyia hominivorax, Coquerel and Its Myiasis. Annal of New York Academy of. Science 23:248-254, 1996.
7. Caiaffa Fllho HH, Mendes CM. Identificação das bactérias Gram-negativas não fermentadoras da glicose ( $1^{\underline{a}}$ de 2 partes). Revista Brasileira de Patologia Clinica 22:213221, 1986.

8. Caiaffa Filho HH, Mendes $\mathrm{CM}$. Identificação das bactérias Gram-negativas não fermentadoras da glicose ( $2^{\mathrm{a}}$ de 2 partes). Revista Brasileira de Patologia Clínica 23:1217, 1987.

9. Cameron MM, Milligan PJM, Llanoscuenta AS, Davis CR. An association between phlebotomine sandflies and aphids in the Peruvian Andes Medical and Veterinary Entomology 9:127-132, 1995a.

10. Cameron MM, Pessoa FA, Vasconcelos AW, Ward RD. Sugar meal sources for the phlebotomine sandflies Lutzomyia longipalpis in Ceará State, Brazil. Medical and Veterinary Entomology 9:263-272, 1995b.

11. Demaio J, Pumpuni CB, Kent M, Beier JC. The bacterial flora of wild Aedes triseriatus, Culex pipiens and Psorophora columbiae mosquitos. American Journal of Tropical Medicine and Hygiene 54:219-223, 1996.

12. Dillon RJ, El Kordy E, Lane RP. The prevalence of microbiota in the digestive tract of Phlebotomus papatasi. Annals of Tropical Medicine and Parasitology 90:669-673, 1996. 
13. Figueiredo AR. Isolamento da microbiota bacteriana de triatomíneos e persistência do Enterobacter cloacae em Rhodnius prolixus Stal, 1859. Tese de Mestrado. Fundação Oswaldo Cruz, Rio de Janeiro, RJ 1-72 1995.

14. Figueiredo AR, Oliveira SMPde, Gordano-dias CM, d'Almeida JM, Brazil RP. Presença de Serratia marcescens e Pseudomonas aeruginosa em colônias de flebotomíneos (Diptera: Psychodidae). 16ํㅡㄹ Congresso Brasileiro de Entomologia. VII ENFIT- Encontro Nacional de Fitossanitaristas. Salvador -BA 1997.

15. Holt JG, Krieg NR, Sneath PHA, Staley JT, Williams ST. Bergey's Manual of deteminative bacteriology. $9^{\text {th }}$ edition. Williams \& Wilkins, Baltimore, 1996.

16. Jadin J. Du role des bacteries dans le tube digestif des insectes vecteurs des plasmodidae et des Trypanosomidae. Annales de Societé Belgique de Medicine Tropicale 47:331-342, 1967

17. Killick- Kendrick R, Killick- Kendrick M. Honeydew of aphids as a source of sugar for Phlebotomus ariasi. Medical and Veterinary Entomology 1:297-302, 1987.

18. Koneman EW, Allen SD, Dowel JRV, Sommes HM. Diagnóstico microbiológico: Texto e bas bioquímicas para la identificatión de bactérias de importancia clínica. $2^{a}$ edição. Panamericana, São Paulo, 1993.

19. Lacey LA, Federicl BA. Pathogenesis and midgut histopathology of Bacillus thuringiensis in Simulium vittatum (Diptera: Simuliidae). Journal of Invertebrate Pathology 33: 171-182 1979.

20.Lacey LA, Undeen AH. Microbial control of black flies and mosquitoes. Annual Review of Entomology 31: 265-296 1986.

21.Lennette EH, Balows A, Shadomy HJ. Mannual of clinical microbiology. $4^{\text {th }}$ edition, ASM Press, Washington, DC, 1985.

22.Mac-Fadin A. Pruebas bioquímicas para la identificatión de bactérias de importancia clínica. $2^{a}$ edição. Panamericana, São Paulo, 1993.

23. Moore GF. Mortality Factors Caused by Pathogenic Bacteria and Fungi of the Southern Pinee Beetle in North Carolina. Journal of Invertebrate Pathology 17:28-37, 1971.

24. Oliveira SMP, Loureiro MM, Leandro MJF, Giordano-Dias CM, Brazil RP, d'Almeida JM, Asensi MD, Mello RP.
Caracterização da microbiota do trato digestivo de fêmeas de Lutzomyia longipalpis (Lutz \& Neiva, 1912) (Diptera: Psychodidae) alimentadas com frutose. Entomologia y Vectores 6:166-179, 1999.

25. Pell PE, Southern DI. Symbionts in the Female Tsetse Fly Glossina morsitans morsitans. Experientia 31:650651, 1975.

26. Pumpuni CB, Demaio J, Kent M, Davis JR, Beier JC. Bacterial population Dynamics in three Anopheline species: The impact on Plasmodium sporogonic development. American Journal of Tropical Medicine and Hygiene 54:214-218, 1996.

27. Schlein Y, Muller G. Assessment of plant tissue feeeding by sandflies (Diptera: Psychodidae) and mosquitos (Diptera: Culicidae). Journal of Medical Entomology 32:882-888, 1995.

28. Schlein Y, Yuval B. Leishmaniasis in the Jordan Valley. IV. Attraction of Phlebotomus papatasi (Diptera: Psychodidae) to plants in the field. Journal of Medical Entomology 24:87-90, 1987.

29. Schlein Y, Polacheck, Yuval B. Mycoses, bacterial infection and antibacterial activity in sandflies (Psychodidae) and their possible role in the transmission of leishmaniasis. Parasitology 90:57-66, 1985.

30. Seitz HM, Maier WA, Rottok M, Becker-Feldmann H. Concomitant infections of Anopheles stephensi with Plasmodium berghei and Serratia mascescens: additive detrimental effects. Zentralbl Bakt Hygiene 266:155-166, 1987.

31. Szabó I, Marton M, Buti I, Pártai G. Intestinal microflora of the larvae of St.Mark's fly. Acta Microbiological Academic Sienci Hung.13:47-52, 1966.

32. Tanada Y, Kaya HKI. Insect Pathology. Academic Press. New York. 12-51, 1993.

33. Yuval B, Warburg A. Susceptibility of adult phlebotomine sandflies (Diptera: Psychodidae) to Bacillus thuringiensis var. israeliensis. Annals of Tropical Medicine and Parasitology 83:195-196, 1989.

34. Yuval B, Warburg A, Schlein. Leishmaniasis in the Jordan Valley. V. Dispersal characteristics of the sandfly Phlebotomus papatasi. Medical and Veterinary Entomology 2:391-395, 1988. 